\title{
Role of free radicals and antioxidants in cervical cancer in southern part of Rajasthan
}

\author{
Priyanka $^{1, *}$, Dharmveer $^{2}$, Vinay $^{3}$ \\ ${ }^{1}$ Senior Demonstrator, Dept. of Biochemistry, Rabindranath Tagore Medical College, Udaipur Rajasthan, ${ }^{2}$ Medical Officer, \\ Sardar Patel Medical College, Bikaner, Rajasthan, ${ }^{3}$ Senior Demonstrator, Geetanjali Medical College, Udaipur Rajasthan, India \\ *Corresponding Author: Priyanka \\ Email: priyanka.mali87@gmail.com
}

Received: $30^{\text {th }}$ May, 2018

Accepted: $15^{\text {th }}$ June, 2018

\begin{abstract}
Introduction: Present study is conducted to measure the role of free radicals malondialdehyde and vitamin-C and vitamin-E in cervical cancer women in southern part of Rajasthan.

Materials and Methods: Total 80 cases and controls were taken and level of MDA were determined by the Thiobarbituric acid (TBA) test which is the colorimetric reaction of MDA and TBA in acid solution. Vitamin $\mathrm{C}$ was determined by the method of Natelson, (1971). Vitamin E was determined by Baker and Frank method.

Results: The serum level of malondialdehyde, vitamin- $\mathrm{C}$ and vitamin-E level in cervix cancer patients and controls using paired t-test for independent means and found that a statistically significant difference exist between case and controls.

Conclusion: Present study concluded that serum levels of vitamin $C$ and vitamin E decreases and level of MDA increases in cervix cancer patients. So the serum levels of these micronutrients as a marker in early diagnosis of cervix cancer as adjuvant to other methods as well as by providing supplementation of these micronutrients.
\end{abstract}

Keywords: Cervix carcinoma, Malondialdehyde, Oxidative stress, Polyunsaturated fatty acid, Thiobarbituric acid reactive substances, Antioxidant vitamins.

\section{Introduction}

Cancer is a foremost cause of mortality in both more or less economically developed nations. According to statistical profile in 2012, cervix cancer in the fourth most common cancer worldwide and in developed nations. In evolving countries cervical cancer is the $2^{\text {nd }}$ most common cancer in females (Lindsey et al., 2015). In India every year 122,844 women are diagnosed with cervix cancer and 67,477 women die. It is the second most common cancer in women aged 15-44 years. India also has the highest age standardized incidence of cervical cancer in South Asia at 22, compared to 19.2 in Bangladesh, 13 in Sri Lanka, and 2.8 in Iran.5 Therefore, it is vital to understand the epidemiology of cervical cancer in India (Sreedevi et al., 2016).

Cervical cancer tends to occur in mid age of women, with $50 \%$ of the cancer patients diagnosed between 25 to 65 years of age. Cervix cancer hardly affects women under the age of 20 (Naidu et al, 2007). Most contributing epidemiological risk factors are: early age sexual intercourse, use of oral contraceptive pills, sexual promiscuity, and human papillomavirus (HPV) infection (Gonçalves et al, 2005). The incidence of cervix infection with human papillomavirus (HPV) in women varies greatly worldwide, and is closely associated with corresponding risk of cervical cancer (Clifford et al., 2005).

Oxidative stress plays an important role in the development of several human disease. These comprises cancer, atherosclerosis, diabetes, cardiovascular disease, neurodegenerative diseases, pulmonary fibrosis, liver diseases, AIDS and aging (Waris and Ahsan, 2006). Free radical induces peroxidation of membrane lipids. As a results their occur alteration in the structural and functional organization of the cell membrane (Manoharan et al, 2004).

For the maintenance of normal cell functioning and ensuring cell survival the regulation of redox homeostasis is essential. An elevated level of reactive oxygen species in cancer cells is an outcome of altered signalling pathways that affects metabolism of cells. Malondialdehyde (MDA) and 4-hydroxynonenal (4HNE) are the most common reactive species of membrane lipid peroxidation, and have been established to play a crucial role in the development and pathogenesis of many disorders and inflammatory reactions (Manoharan et al, 2004). 4-HNE appears to be the most toxic product of lipid peroxidation whereas MDA is the most mutagenic species (Waris and Ahsan, 2006).

By the estimation of stable end product of lipid peroxidation for example MDA we can measure the extent of damage caused by oxidative stress. Level of MDA is commonly used as a marker of oxidative stress and the antioxidant status. (Agarwal et al., 2005).

Recently, use of antioxidant vitamins have received much attention in accordance to prevention of cancer, chiefly because of their role in neutralization of free radicals and oxidants and thus prevent free-radical damage to DNA; enhance the immune system (Guo et al, 2015). Tocopherols (vitamin E), ascorbic acid (vitamin C), and the carotenoids react with free 
radicals, notably peroxyl radicals, and with singlet molecular oxygen, which is the basis for their function as antioxidants. RRR-alpha-Tocopherol is the major peroxyl radical scavenger in biological lipid phases such as membranes or low-density lipoproteins. Few studies reveal that use of vitamin $\mathrm{C}$ reduces risk of cancer by providing a degree of protection to DNA damage by reactive oxygen species (Goncalves et al, 2005). The function of vitamin $C$ as an antioxidant is indicated by its known free radical scavenging activity. Vitamin $\mathrm{C}$ also has a role in sparing vitamin $\mathrm{E}$, which is a lipid soluble antioxidant. The vitamin $\mathrm{C}$ level may control the functioning of vitamin $\mathrm{E}$ as an antioxidant and influence where it gets used. The synergism between vitamins $\mathrm{C}$ and $\mathrm{E}$ results in the more consumption of vitamin $\mathrm{C}$ and the sparing of vitamin $\mathrm{E}$, results in significant decreases in plasma ascorbic acid levels (Kim et al., 2010)

Vitamin $\mathrm{E}$ is a chain terminator lipid soluble antioxidant vitamin found in association with lipids of cell membrane. It functions as cancer inhibitor via its action as an antioxidant vitamin, as well as its potential effects on selenium. It decreases nitrite level, thereby inhibiting the production of carcinogenic nitrous amine and nitrous amides and expression of certain oncogenes (Nimse and Pal, 2015).

The damage to DNA, lipids and proteins is caused by an imbalance between the production and detoxification of free radicals results in oxidative stress. Previous studies show that antioxidant systems are deranged in cancer patients (Demirci et al, 2011). Therefore, the present study was designed to examine the lipid peroxidation status by estimating MDA and the antioxidant status through vitamin $\mathrm{C}$ and vitamin $\mathrm{E}$ in women with cervical cancer.

\section{Materials and Methods}

The study was conducted in the department of Medical biochemistry at Geetanjali medical college \& Hospital. For calculating sample size of 40 we used confidence level of $95 \%$, confidence interval $5 \%$ and population size of 40 we get 37 cases and we consider number of controls also as 40 patients.

Inclusion Criteria: Study included newly diagnosed cervix cancer patient who does not started any treatment (like chemotherapy, radiotherapy etc) attended Geetanjali cancer center in Geetanjali Medical college \& Hospital Udaipur (Raj.).

Exclusion Criteria: The general exclusion criteria were: previous cancer, serious cardiac, Metabolic or liver disease, regular high-dose vitamin supplementation during three months prior to the study or occasional vitamin supplementation during the week prior to inclusion in the study.

$10 \mathrm{ml}$ fasting blood sample was collected in a plain vial by vein puncture and allowed the blood to clot at room temperature and centrifuged at $3000 \mathrm{rpm}$ for 10 min. The serum separated into proper aliquots and analyzed for test. Blood sample was investigated for various parameters like TBARS (MDA) and vitamin C and vitamin $\mathrm{E}$.

Malondialdehyde (MDA) levels were determined by the Thiobarbituric acid (TBA) test which is the colorimetric reaction of MDA and TBA in acid solution. Vitamin $\mathrm{C}$ was determined by the method of Natelson, (1971). Vitamin E was determined by Baker and Frank method.

\section{Results}

A total of 80 cases were studied out of then 40 were cervix cancer patients and 40 were controls. Majority of patients in age group 46-60 years followed by age group 30-45 years. Majority of the patients were belongs to rural area. In our study females suffering from cervix cancer were Hindu, very few were Muslim women. In table 1 we have compared the serum level of MDA, vitamin $\mathrm{E}$ and vitamin $\mathrm{C}$ in cervix cancer patients and controls using paired t-test for independent means and found that a statistically significant difference exist between case and controls.

Table 1: Comparison between cervix cancer patients and controls for serum MDA, vitamin $E$ and vitamin $C$

\begin{tabular}{|c|c|c|c|}
\hline & Mean \pm SD (cases) & Mean \pm SD (Controls) & p-value \\
\hline MDA (n=40) & $7.6358 \pm 2.07$ & $2.5048 \pm 1.09$ & $<0.0001$ \\
\hline Vitamin C (n-40) & $0.5643 \pm 0.266$ & $1.02 \pm 0.187$ & $<0.0001$ \\
\hline Vitamin E $(n=40)$ & $0.51 \pm 0.33$ & $1.04 \pm 0.265$ & $<0.0001$ \\
\hline At confidence level of $95 \%$ & \\
\hline
\end{tabular}

Here, we have compared the serum level of malondialdehyde, vitamin-C and vitamin-E level in cervix cancer patients and controls using paired t-test for independent means and found that a statistically significant difference exist between case and controls. 


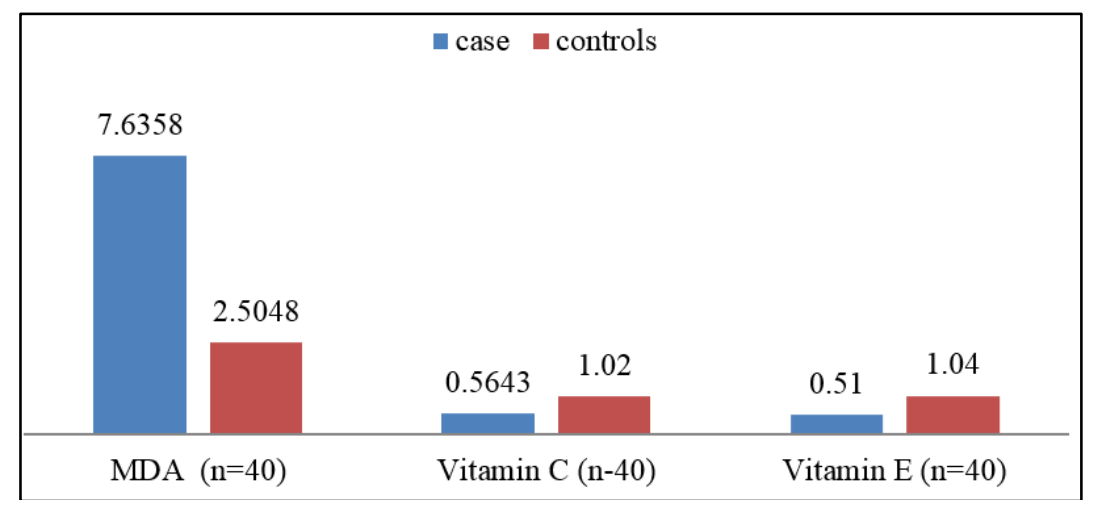

Fig. 1: Comparison between cervix cancer patients and controls for serum MDA, vitamin $E$ and vitamin $C$

We also calculated the Pearson correlation between MDA-vitamin E and vitamin C- vitamin E, but we do not found a significant correlation between these parameters.

Table 2 (a, b) shows the Pearson correlation coefficient (r) between MDA and vitamin-C in cervix cancer patients. There is highly significant negative correlation (-0.796) between MDA and vitamin C level in cases.
Table 2a: Descriptive statistics

\begin{tabular}{|l|c|c|c|}
\hline & Mean & Std. Deviation & n \\
\hline MDA (Cases) & 8.1 & 2.2 & 40 \\
\hline $\begin{array}{l}\text { Vitamin-C } \\
\text { (cases) }\end{array}$ & 0.204 & 0.061 & 40 \\
\hline
\end{tabular}

Table 2b: Correlation values

\begin{tabular}{|l|c|c|}
\hline & $\begin{array}{c}\text { MDA (Cases) } \\
(\mathbf{r})\end{array}$ & $\begin{array}{c}\text { Vitamin-C(cases) } \\
(\mathbf{r})\end{array}$ \\
\hline MDA (Cases) & 1 & -0.796 \\
\hline $\begin{array}{l}\text { Vitamin- } \\
\text { C(cases) }\end{array}$ & -0.796 & 1 \\
\hline \multicolumn{2}{|c|}{ Correlation significant at the level of 0.01 level } \\
\hline
\end{tabular}

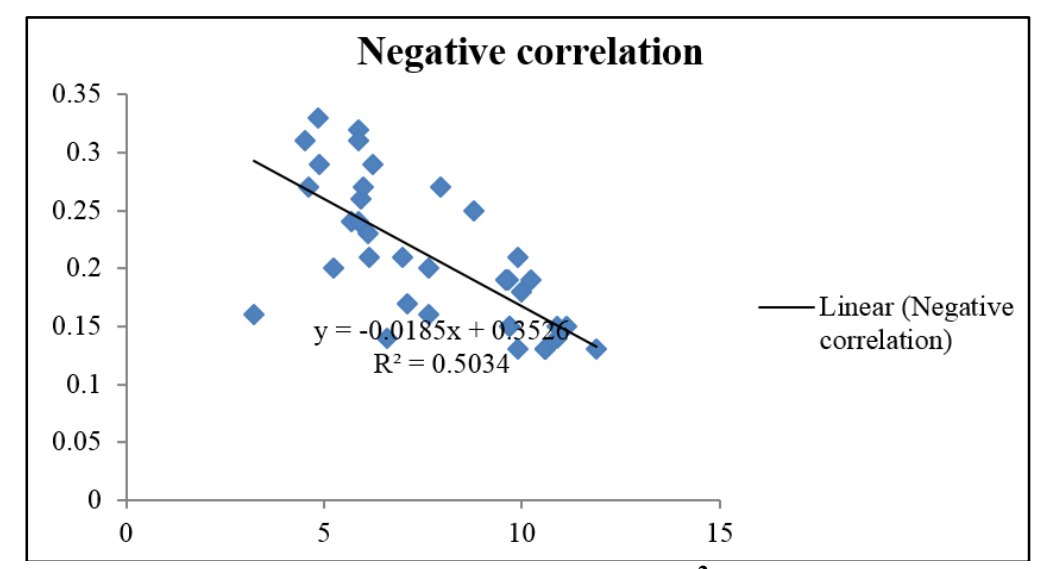

Fig. 2: Showing a negative linear Pearson correlation $\left(r=-0.796 ; R^{2}=0.503\right)$ between $M D A$ and vitamin $C$ in cervix cancer patients

\section{Discussion}

The present study was carried out in oncology and Biochemistry department of Geetanjali Medical College and Hospital and it included a total of 40 newly diagnosed Cervix cancer patients and 40 controls. We had determined the level of MDA, vitamin $\mathrm{C}$ and vitamin $\mathrm{E}$ in the serum of all the patients as well as in controls and finally the results of cervix cancer patients were compared with controls.

Cancer of cervix is a complex and multifactorial disease. Dietary and hematological antioxidants vitamin-E and vitamin-C, might decrease the possibility of cervical dysplasia and cancer (Kim et al, 2010). Goncalves et al conducted a study in which $32.6 \%$ cases were in 20-45 years and $43 \%$ cases were in age group 30-64 years (Goncalves et al, 2005). Similarly, Kim et al, also found higher incidences and prevalence of cervical cancer in age group 30-49 years followed by 50-60 years (Kim et al, 2010).

The present study shows that incidence and mortality is high in rural areas as compared to urban areas. Mallath et al also reported that cancer mortality rates are also significantly high in rural areas, as compared to urban regions, where facilities for cancer treatment are limited. Due to low socioeconomic status, 
ill-literacy, limited health facilities and unawareness in economically poor individuals are at a higher risk of age-specific mortality than affluent people (Mallath et al, 2014).

Free radical induced oxidative stress and deranged antioxidant system have been considered as a potential risk factors involved in the pathophysiology of various disorders, including carcinogenesis. Many studies suggests that women having gynecological disorders have altered antioxidant enzyme activity and it also pointing towards the elevated levels of oxidative stress and lipid peroxidation products (Pejic et al, 2009). The present study shows that serum MDA level is significantly elevated in women with cervical cancer cases. Similarly, other studies also show that increased levels of lipid peroxides in the plasma and erythrocytes were reported for patients with cervical cancer. Ahmed et al. reported significantly elevated levels of lipid peroxidation products in cervical cancer tissues than in normal samples. They also co-related a deranged antioxidant status in cancer of the cervix to tumor development (Manoharan et al, 2004). Lipid peroxidation plays an important role in the pathogenesis of numerous disorders including cancer (Gonclave et al, 2005).

Kierzenkowska et al studied a mutual relationship between free radical activities, free radical scavenging activity of antioxidants, and cervix cancer. They also demonstrated oipaired antioxidant status in cervical cancer. The low activity of antioxidant enzyme may be attributed to reduced synthesis of said enzymes in the cancer tissue. The raised level of products of lipid peroxidation is a result of extensive damage to tissue or a decrease in the efficacy of the antioxidant defense mechanism (Kierzenkowska et al, 2004). In this study, we show that serum vitamin $C$ and vitamin $E$ level is significantly decreased in women with cervical cancer cases.

Similarly, these studies also showed that antioxidant vitamins, such as $\alpha$-carotene, $\beta$-carotene, vitamin $\mathrm{E}$, and vitamin $\mathrm{C}$ could act as efficient scavengers of free radicals and oxidants to prevent freeradical damage to DNA. Furthermore, if an antioxidant enzyme does not neutralize free radicals and reactive oxygen species, inflammatory processes would result in extensive damage to DNA proteins. It has also been formulated that having antioxidant properties may guard the immune system of the body from oxidative stress; improve immune mecahnism (Guo et al, 2015). Antioxidant vitamins are an essential part of human non-enzymatic antioxidant defense (Gonclave et al, 2005).

The decreased levels of vitamin-C may be associated with its action as antioxidant where it gets utilized. Its synergism with vitamin-E helps in sparing of vitamin-E, during this process vitamin-C gets utilized which is seen as significant decline in plasma ascorbic acid. Negative correlation $(r=-0.73)$ between
vitamin-C and MDA was noted leading to the conclusion that free radicals are scavenged by ascorbic acid and thus it gets utilized (Naidu et al, 2007). Ascorbic acid is a part of an aqueous compartment of cell and can decreases the level of tocopherol radical (Sies and Stahl, 1995).It also helps in recycling of oxidized vitamin E (Agarwal et al, 2005) In line with these studies, we also found a negative correlation ( $\mathrm{r}=$ 0.79 ) between MDA and vitamin C (Table:15). We also calculated the Pearson correlation between MDAvitamin $\mathrm{E}$ and vitamin $\mathrm{C}$ - vitamin $\mathrm{E}$, but we do not found a significant correlation between these parameters.

The results of the present study suggest that vitamin $\mathrm{C}$ and vitamin $\mathrm{E}$ can be considered a biochemical marker of oxidative stress associated with cancer and can be recommended to patients to check or slowing down the progression of cervical cancer. The etiology of cervical cancer is multifactorial and various risk factors have been described. Deficiency of nutrients in developing countries can be a significant contributing factor in modifying the multistage process of carcinogenesis.

\section{Conclusion}

Cervix cancer is a major cause of morbidity and mortality in women of both developing and developed countries. Though many advancements has been done to improve early diagnosis and proper management of cervix cancer, still the increasing incidence of cervix cancer demands some new reliable and easy method for detection of cervix cancer. Present study concluded that serum levels of vitamin $\mathrm{C}$ and vitamin $\mathrm{E}$ decreases and level of MDA increases in cervix cancer patients. So the serum levels of these micronutrients as a marker in early diagnosis of cervix cancer as adjuvant to other methods as well as by providing supplementation of these micronutrients. Thus, present study can be helpful in improving the prognosis of patient.

\section{References}

1. Agarwal A. Gupta S. and Sharma RK. "Role of oxidative stress in female reproduction" Reproductive Biology and Endocrinology. 2005;3:1477-7827.

2. Clifford GM. Gallus S. Herrero R. Muñoz H. Snijders PJ. Vaccarella S. Ferreccio C. Hieu NT. Matos E. Molano M. Rajkumar R. Ronco G. "Worldwide distribution of human papillomavirus types in cytologically normal women in the International Agency for Research on Cancer HPV prevalence surveys." Lancet. 2005;366:99198.

3. Demirci S. Ozsaran Z. Celik HA. Aras AB. and Aydin HH. "The interaction between antioxidant status and cervical cancer: a case control study." Tumori 2011;97:290-295.

4. Dikshit R. Gupta PC. Ramasundarahettige C. Gajalakshmi V. Aleksandrowicz L. Badwe R. "Cancer mortality in India: a nationally representative survey." (2012) DOI:10.1016/S0140-6736(12)60358-4 
5. Gonçalves TL, Cristiane EF, Müller LG, Piovezan CM, Nogueira CW and Rocha JBT. "Involvement of oxidative stress in the pre-malignant and malignant states of cervical cancer in women." Clinical Biochemistry. 2005;38:1071-1075.

6. Guo L. Zhu H. Lin C. Che J. Tian X. Han S. Zhao H. Zhu Y. and Mao D. "Associations between antioxidant vitamins and the risk of invasive cervical cancer in Chinese women. A case-control study." Scientific Reports. 2015;5:13607.

7. Kierzenkowska CM, Kornelia Kedziora kornatowska, Wozniak, Tomaszdrewa, Wozniak B. and Makarewicz. "The effect of brachytherapy on Antioxidant Status and Lipid Peroxidation in patients with cancer of the uterine cervix." Cellular and Molecular Biology Letters. 2004;9:511-518.

8. Kim J, Kim MK, Lee JK, Kim JH, Son SK, Song ES, et al. "Intakes of Vitamin A, C, and E, and $\beta$-Carotene Are Associated With Risk of Cervical Cancer." A CaseControl Study in Korea Nutrition and Cancer. 2010;62:181-189.

9. Lindsey A, Torre, Bray F, Rebecca L, Ferlay J, Tieulent JL, Jemal A. "Global Cancer Statistics." Ca Cancer J Clin. 2015;65:87-108.

10. Mallath MK. Taylor DG. Badwe RA. Rath GK. Shanta V. Pramesh CS. Digumart R. Sebastian P. "The growing burden of cancer in India: epidemiology and social context" (2014); http://dx.doi.org/10.1016/S14702045(14)70115-9

11. Manoharan S. Kolanjiappan K. and Kayalvizhi MK. "Enhanced Lipid Peroxidation and Impaired Enzymic Antioxidant Activities in the Erythrocytes of patients with Cervical Carcinoma." Cellular and Molicular Biology Litters. 2004;9:699-707.
12. Naidu MS. Suryakar AN. Swami SC. Katkam RV, and Kumbar K. "Oxidative stress and Antioxidant Status in Cervical Cancer Patients." Indian Journal of Clinical Biochemistry. 2007;22:140-144.

13. Nimse SB. and Palb D. "Free radicals, natural antioxidants, and their reaction mechanisms." Royal Society Chemistry Advances. 2015;5:27986.

14. Pejić S. Todorović A. Stojiljković V. Kasapović J. and Pajović SB. "Antioxidant enzymes and lipid peroxidation in endometrium of patients with polyps, myoma, hyperplasia and adenocarcinoma." Reproductive Biology and Endocrinology. 2009;7:149.

15. Sies H. and Stahl W. "Vitamins E and C, beta-carotene, and other carotenoids as antioxidants" American Journal of Clinical Nutrition. 1995;62:1315-1321.

16. Sreedevi A, Javed R, Dinesh A. "Epidemiology of cervical cancer with special focus on India." International Journal of Womens Health. 2015;7:405-17.

17. Waris G. and Ahsan H. Reactive oxygen species: role in the development of cancer and various chronic conditions." Journal of Carcinogenesis. 2006;5:14.

How to cite this article: Priyanka, Dharmveer, Vinay. Role of free radicals and antioxidants in cervical cancer in southern part of Rajasthan. Int J Clin Biochem Res. 2018;5(4):555-559. 